\title{
Arterial blood gas analysis: as safe as we think? A multicentre historical cohort study
}

\author{
Sacha C. Rowling $\mathbb{\mathbb { D }}^{1,2}$, Marianne Fløjstrup $\mathbb{1}^{1,3}$, Daniel Pilsgaard Henriksen $\mathbb{\mathbb { D }}^{1}$, Bjarke Viberg $\mathbb{\mathbb { D }}^{4}$,

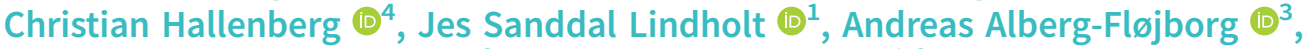 \\ Prabath W.B. Nanayakkara (10) ${ }^{2}$ and Mikkel Brabrand (1) ${ }^{1,3}$
}

${ }^{1}$ Odense University Hospital, University of Southern Denmark, Odense, Denmark. ${ }^{2}$ Amsterdam University Medical Center, Location VU University Medical Centre, Vrije Universiteit Amsterdam, Amsterdam, The Netherlands. ${ }^{3}$ Hospital of South West Jutland, Esbjerg, Denmark. ${ }^{4}$ Hospital Lillebaelt, University Hospital of Southern Denmark, Vejle, Denmark.

Corresponding author: Sacha C. Rowling (sacharowling@live.nl)

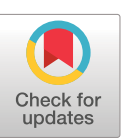

This version is distributed under the terms of the Creative Commons Attribution Non-Commercial Licence 4.0. For commercial reproduction rights and permissions contact permissions@ersnet.org

Received: 1 Sept 2021 Accepted: 4 Jan 2022

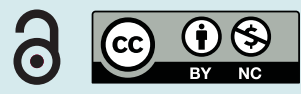

Shareable abstract (@ERSpublications)

Arterial punctures for arterial blood gas analysis are safe procedures with a major complication rate within 7 days of $0.14 \%(95 \% \mathrm{Cl} 0.13-0.15 \%)$. Patients on antithrombotic medication have an increased risk of developing major complications. https://bit.ly/3FaPOwk

Cite this article as: Rowling SC, Fløjstrup M, Henriksen DP, et al. Arterial blood gas analysis: as safe as we think? A multicentre historical cohort study. ERJ Open Res 2022; 8: 00535-2021 [DOI: 10.1183/ 23120541.00535-2021].

\section{Abstract}

Purpose Arterial punctures (APs) for arterial blood gas (ABG) analyses are much-used medical procedures. To date, no large studies have been conducted on the major complication rate of APs. We aimed to describe the risk of major complications within 7 days after puncture and investigate whether using antithrombotic medication affected this.

Methods We included all APs performed for ABG analysis at three Danish hospitals from January 1, 1993 to February 25, 2013. We excluded APs ordered by the anaesthesiology department, intensive care unit (ICU) or in patients $<18$ years old. Data on the patient level were extracted from the Danish National Patient Registry, Danish Civil Registration System and Odense Pharmaco-Epidemiologic Database (OPED), the latter providing us with information on antithrombotic medication. Initially, two clinicians compiled a list with all procedures and diagnoses that could possibly be a consequence of APs. The selected procedures and diagnoses were further categorised independently by three surgeons and used to indicate the complication rate.

Results We analysed 473327 APs and found 669 (0.14\%, 95\% CI 0.13-0.15) APs led to major complications: embolisms or thrombosis (49.0\%), aneurysms (15.4\%), nerve damage (1.5\%), arteriovenous fistulas $(0.6 \%)$ or of another kind (33.5\%). The identified major complication rates in patients on antithrombotic medication were increased (OR 1.31, 95\% CI 1.07-1.61).

Conclusion APs for ABG analyses are safe procedures. The major complication rate within 7 days was 0.14\% (95\% CI 0.13-0.15). Patients on antithrombotic medication carry an increased risk of developing major complications.

\section{Introduction}

Sampling for arterial blood gas (ABG) analysis is a well-known and much-used invasive procedure applied within hospital departments on a daily basis. It is of importance in diagnosing and assessing acutely ill patients, easy to perform and can be executed by doctors, nurses and laboratory technicians [1, 2]. In addition, sampling is possibly painful and distressing because of the highly innervated sampling locations and occasional multiple attempts [3-5]. Contraindications are relative and strict and a standard protocol should be followed, all with the aim of reducing technical difficulties and possible harm done to the patient $[6,7]$.

The assumption that arterial punctures (APs) are safe is generally based on small, fairly out-dated studies that mainly focus on minor complications. For example, 4342 APs obtained in a military setting from 1969 
to 1970 resulted in 25 haematomas (0.58\%), none of which were defined as a major complication [8]. A study using computed tomography scans revealed haematoma formation in 128 out of 270 patients (47.4\%), none of which progressed to a severe condition [9]. Another study of 6185 brachial artery punctures found an overall incidence of complications of $2.0 \%$, varying from immediate limb pain or paraesthesia to haematoma formation. Again, none of the complications were considered to be major [10]. All of the previously found minor complications resolved spontaneously [8-10]. Major complications have generally been defined as leading to prolonged hospitalisation time or a need for surgical treatment or another intervention due to ischaemia or other objective abnormalities [8-12]. Described major complications include thrombosis with distal ischaemia, major haemorrhages and haematomas, (pseudo) aneurysms, nerve damage and arteriovenous fistulas. The largest published study, reporting numbers on these major complications, included 266 punctures: the previously mentioned major complications occurred in $3.6 \%$ to $10.3 \%$ of the APs [11]. A study from 1967 found that 19 out of 1466 (1.3\%) percutaneous needle punctures led to major complications, thrombosis at the entry site with distal ischaemia being the most common [12]. Similar and other unique complications such as an arteriovenous fistula leading to pulmonary oedema and a small bowel perforation due to femoral herniation have been reported in a number of case reports [13-20].

Overall, major complications can occur, but studies show variety in the incidence of complications, and sample sizes are too small to make inferences about the occurrence of the different types of major complications. Although complications may be limited, a serious complication such as thrombosis may have dire consequences for the patient. This particular topic needs to be studied in further detail. An AP is a routinely performed procedure and complications are not taken into consideration, since it is unclear what the consequences are and at what rates they occur. In addition, little is known about the risk of major complications in patients on antithrombotic medication [12, 19, 21, 22].

The primary aim of this paper is to assess the incidence of major complications, 7 days after APs for ABG analyses. Our secondary aim is to analyse whether patients on antithrombotic medication prior to hospitalisation have a higher complication rate. Our hypothesis is that there is a very low major complication rate within 7 days after arterial blood sampling for an ABG. We expect the major complication rate to be comparable in patients on and off antithrombotic medication.

Methods

This paper is reported in conjunction with the RECORD Guidelines [23]. This study was approved by the Danish Data Protection Agency (approval number 2008-58-0035) and the Danish National Board of Health (approval number 3-3013-122/1). The regional Ethics Committee on science confirmed that the project did not require approval according to Danish law.

\section{Study design}

We performed a multicentre historical cohort study with prospectively collected data that were analysed retrospectively.

\section{Setting}

Data were collected from three hospitals in the region of Southern Denmark (Hospital of South West Jutland; Hospital Lillebaelt; Odense University Hospital) on all ABGs between January 1, 1993 and February 25, 2013.

\section{Participants}

All adult patients ( $>18$ years) with a Danish unique identifying “Central Persons Register” (CPR) number with a registered $A B G$ within the study period were included. APs were excluded if the ABG was ordered by the intensive care unit (ICU) or the anaesthesiology department, because these samples were possibly drawn from indwelling arterial catheters. Since this study had a short follow-up time, we assumed no patients emigrated within 7 days after the AP and therefore did not exclude patients based on this element.

\section{Variables}

The variables used in this study on patient level were age, sex and Charlson Comorbidity Index (CCI), the latter categorised as CCI score of $0,1,2$ or $>3$. The CCI was used to describe the degree of comorbidity in patients $[24,25]$. This study analysed every puncture separately because we wanted to know how many APs led to a major complication. The primary outcome was the occurrence of major complications within 7 days after the AP, which were defined as a procedure or diagnosis registered in the national registries with respectively Sundheds-væsenets Klassifikations System (SKS) or International Classification of 
Diseases (ICD) codes that could have been the result (i.e. a proxy) of the complication. The secondary outcome was the major complication rate in patients on antithrombotic medication.

\section{Data sources/measurement}

Data on patient level were extracted from the Danish National Patient Registry, the Danish Civil Registration System and the Odense Pharmaco-Epidemiologic Database (OPED). Information on prior, current and later hospitalisations was requested from the Danish National Patient Registry and follow-up information on vital status from the Danish Civil Registration System [26, 27]. All the registered procedures and diagnoses in the patients who had an ABG ordered were listed. This list included both SKS codes (NOMESCO codes Scandinavian standard procedure codes) and ICD-8 and ICD-10 codes (codes for diagnoses) [27]. SKS codes are registered at the time of the procedure; ICD codes are registered at the time of transfer between wards or at hospital discharge. Information about prescribed antithrombotic medication (anticoagulant and antiplatelet medication) prior to hospitalisation was received from the OPED, which was used to register patients who had reimbursed a prescription within 90 days before the AP [28, 29].

\section{Bias}

A description of the efforts made to address potential sources of bias is described under the heading Quantitative variables.

\section{Study size}

The study size was arrived at by including all available data on our topic of interest within the study period.

\section{Quantitative variables}

Two of the authors - a cardiologist and a clinical pharmacologist (M.B. and D.P.H.) - independently went through the list of SKS, ICD-8 and ICD-10 codes and excluded all procedures and diagnoses that were clearly irrelevant to our study (e.g. caesarean section or hip replacement), resolving disagreement by oral consensus. To create the most accurate description of possible complications, three independently blinded surgeons - one orthopaedic surgeon (B.V.) and two vascular surgeons (C.H. and J.S.L.) - assessed the selected procedures and diagnoses and classified them as "Very likely", "Possible but unlikely" and "Not possible" to be the result of an AP (see table 1 for a list of all the SKS, ICD-8 and ICD-10 codes that were included by the two authors, and how they were categorised by the surgeons). In the analyses, all the procedures or diagnoses that were marked by at least one of the surgeons as "Very likely" were included as being a "major complication", thus giving the realistic complication rate. All procedures and diagnoses marked as "Very likely" or "Possible but unlikely" were included to indicate the maximum complication rate. Because both SKS and ICD codes were used, codes registering the same (type of) complication were clustered through assessment by the two authors: five major complication categories were identified, as shown in table 2.

\section{Statistical methods}

Statistical analysis was performed using Stata/IC 15.0 (Stata Corp, College Station, TX, USA). Data are presented as medians (range) or proportions (95\% confidence interval), since the data were not with a normal distribution. The effect of sex, age, use of antithrombotic medication, CCI and number of prior APs was examined in an unmatched logistic regression and shown with adjusted and unadjusted odds ratios. If information on the CCI was missing, we assumed that the patient did not have any relevant comorbidity. We counted the number of APs in the 7 days prior to the AP that we assumed led to a complication. We only used the last AP on that day. To describe the effect of usage of antithrombotic medication prior to receiving an AP, a chi-square test was used.

\section{Data access and clearing methods}

All authors had full access to the database population which was used to create the study population. As data were extracted from national databases, no additional cleaning was required.

\section{Linkage}

Denmark has unique identifying numbers - Central Persons Register (CPR) numbers - making complete follow-up and true population-based studies possible [26, 27]. The Danish unique identifying CPR numbers were used to link the available data from the multiple databases used within this study.

\section{Results}

Demographics

From January 1, 1993 to February 25, 2013, 975360 ABGs were ordered in the three participating hospitals. We included a total of 473327 APs (figure 1), which were performed in 109696 patients. Some 
TABLE 1 List of Sundheds-væsenets Klassifikations System, International Classification of Diseases-8 and -10 codes that are possibly related to an arterial puncture, as selected by two clinical physicians and categorised by three independent blinded surgeons

Code

Very likely Possible but unlikely Not possible

44299 - Aneurysm arteria aliud

44441 - Embolism, thrombosis arteria femoralis

44442 - Embolism, thrombosis arteria popliteae

44444 - Embolism, thrombosis arteria periphericae extremitatis

68229 - Inflammation, abscess and acute lymphangioma. brachii and antebrachii

95209 - Traumatic lesion plexus brachialis and nervi brachii, uncomplicated

DG560 - Carpal tunnel syndrome

DG562 - Neuropathy in nervus ulnaris

DI721 - Aneurysm upper extremity

DI721 - Aneurysm in artery in upper extremity

DI724 - Aneurysm in artery in lower extremity

DI729 - Aneurysm unspecified

DI742 - Embolism or thrombosis in artery in upper extremity

DI742A - Embolism or thrombosis in upper extremity

DI742A - Emboli arteria brachialis

DI742B - Thrombosis in artery in upper extremity

DI742B - Thrombosis arteria brachialis

DI743 - Embolism or thrombosis in artery in lower extremity

DI743A - Embolism in artery in lower extremity

DI743B - Thrombosis in artery in lower extremity

DI744 - Embolism or thrombosis in artery in unspecified extremity

DI748 - Embolism or thrombosis in other artery

DI749 - Embolism or thrombosis in artery unspecified

DI770 - Acquired A-V fistula

DI771 - Artery stricture

DI771 - Stricture of artery

DI772 - Artery rupture

DI775 - Artery necrosis

DI803A - Embolism in lower extremity unspecified

$x-x x$

DM622A - Non-traumatic compartment syndrome

$\mathrm{X}$

$x$

$X$

DS451 - Lesion of arteria brachialis

DS540 - Lesion of nervus ulnaris in elbow region or lower arm

DS541 - Lesion of nervus medianus in elbow region or lower arm

DS542 - Lesion of nervus radialis in elbow region or lower arm

DS550 - Lesion of nervus ulnaris in elbow region or lower arm

$x$

$x$

$x$

$\begin{array}{cc}x x & x \\ x & \\ x x & \end{array}$

$x x$

$X X$

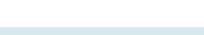

$\begin{array}{ll}x & x \\ x & x\end{array}$

$x x$

$x x$

$x X$

$x$

$X X$

$x$

DS551 - Lesion of arteria radialis in elbow region or lower arm

$x$

$x$

$x \times$

$x$

$x$

$\mathrm{XX}$

DS561 - Lesion of muscle/tendon of long flexor to a finger on lower arm

DS562 - Lesion of muscle or tendon of other flexor on lower arm

DS568 - Lesion of an unspecified muscle or tendon in elbow/lower arm

DS641 - Lesion of nervus medianus in wrist or hand

DS659 - Lesion of blood vessel in wrist or hand unspecified

DS660 - Lesion of long flexors to thumb in wrist/hand

DS661 - Lesion of flexors to unspecified finger in wrist or hand

DS750 - Lesion in arteria femoralis

DT145 - Lesion in blood vessel without indication of body region

DT801 - Complication in vessel after infusion, transfusion on injection

DT802 - Infection after infusion, transfusion or injection

DT808 - Other complication after infusion, transfusion or injection

DT809 - Complication after infusion, transfusion or injection unspecified

DT812K - Inadverted preoperative puncture/lesion of vein or lymph system

DT814 - Infection after intervention unspecified

DT817 - Complication in vessel after intervention unspecified $X$

DT817B - Thromboembolic complication unspecified

DT817K - Thrombosis, embolism or necrosis after vessel operation

DT819 - Complication of procedure unspecified

DT88 - Complications from surgery

DT888 - Complication with surgery or with unspecified treatment, other specified

KACA11 - Exploration, n. medianus

\begin{tabular}{|c|c|c|}
\hline & $x$ & $X X$ \\
\hline & $x$ & $X X$ \\
\hline & & $X X X$ \\
\hline \multirow[t]{2}{*}{$X X$} & & $X$ \\
\hline & $x$ & $X X$ \\
\hline$X$ & $X$ & $X$ \\
\hline$x$ & & $X X$ \\
\hline$X X$ & $x$ & \\
\hline \multicolumn{3}{|c|}{$x \times x$} \\
\hline & $X$ & $X X$ \\
\hline & $X$ & $X X$ \\
\hline & $X$ & $X X$ \\
\hline$X$ & $x$ & $x$ \\
\hline \multirow[t]{3}{*}{$x x$} & & $X$ \\
\hline & $x$ & $X X$ \\
\hline & $x$ & $X X$ \\
\hline$x$ & & $X X$ \\
\hline$x$ & $X$ & $X$ \\
\hline$X X$ & & $X$ \\
\hline$X X$ & & $x$ \\
\hline$X X$ & & $x$ \\
\hline$X X$ & & $X$ \\
\hline \multirow[t]{2}{*}{$x$} & $X$ & $X$ \\
\hline & $X X$ & $X$ \\
\hline \multirow[t]{4}{*}{$X$} & $X X$ & \\
\hline & $X X$ & $x$ \\
\hline & & $X X X$ \\
\hline & $X$ & $X X$ \\
\hline$X$ & $x$ & $x$ \\
\hline \multirow[t]{2}{*}{$X$} & $X X$ & \\
\hline & $X$ & $X X$ \\
\hline
\end{tabular}


TABLE 1 Continued

Code Very likely Possible but unlikely

Not possible

KACA13 - Exploration, n. ulnaris

KACC51 - Decompression and loosening of nervus medianus

KACC53 - Decompression and loosening of nervus ulnaris

KNCA00 - Exploration in soft tissue in lower arm, percutaneous

KNCA02 - Exploration in soft tissue in lower arm, open

KNCM09 - Fasciotomy in lower arm

KNCM19 - Resection or excision of fasciae in lower arm

KNCM99 - Operation on fasciae, tendons, ganglion, bursa, elbow/lower arm, other

KNCS09 - Incision/revision of infection in tendon in elbow/lower arm

KNCS99 - Operation for infection in tendons, joints and bones, elbow/lower arm, other

KNDA02 - Exploration of soft tissue, wrist and hand, open

KNDM09 - Fasciotomy of wrist/hand

KNDM19 - Fasciotomy partial/total (incl. op pro mb. Dupuytren)

KNDS09 - Incision/revision of infection in tendon in wrist/hand

KNDS99 - Other operation for infection in tendon, joint or bone in wrist/hand

KQCA00 - Puncture of skin in upper extremity

KQCA10 - Incision of skin in upper extremity

KQCA99 - Incision of skin in upper extremity, other

KQCBO0 - Suture of skin in upper extremity

KQCB05 - Wound revision in upper extremity

KQCB10 - Replacement of larger wounds in upper extremity

KQCB99 - Wound treatment in upper extremity, other

KTNCOO - Puncture or needle biopsy in elbow/lower arm

KTNC05 - Minor incision or soft tissue in elbow/lower arm

KTND00 - Puncture or needle biopsy in wrist/hand

KTND05 - Minor incision of soft tissue in wrist/hand

$x$
$x x$
$x x$
$x$
$x$
$x x$

$x x$

$x$

$x$

$X X$

$X X$ $X X X$

$X X X$

$\begin{array}{ll} & X X X \\ X & X X\end{array}$

$x \quad x x$

$\mathrm{X}$

$\begin{array}{cc}x & X X X \\ x & X X\end{array}$

$x$

$x X$

$X X$

$X X X$

$X X X$

$\mathrm{XXX}$

$X X X$

$X X$

$\begin{array}{cc}X & X X X \\ X & X X\end{array}$

$x \quad x x$

$x$

$x$

$x x$

$x$

$\mathrm{XX}$

$X X$

$x$

$X X$

TABLE 2 List of Sundheds-væsenets Klassifikations System, International Classification of Diseases-8 and -10 codes that were registered in patients that had a complication after an arterial puncture, categorised into five categories

Code

Category

DI742 Embolism or thrombosis in artery in upper extremity

DI743 Embolism or thrombosis in artery in lower extremity

DI743A Embolism in artery in lower extremity

DI743B Thrombosis in artery in lower extremity

DI744 Embolism or thrombosis in artery in unspecified extremity

DI749 Embolism or thrombosis in artery unspecified

44441 Embolism, thrombosis arteria femoralis

44442 Embolism, thrombosis arteria popliteae

44444 Embolism, thrombosis arteria periphericae extremitatis

DI721 Aneurysm upper extremity

DI721 Aneurysm in artery in upper extremity

DI724 Aneurysm in artery in lower extremity

DI729 Aneurysm unspecified

44299 Aneurysm arteria aliud

DS541 Lesion of nervus medianus in elbow region or lower arm

DS641 Lesion of nervus medianus in wrist or hand

DI770 Acquired A-V fistula

DS550 Lesion of nervus ulnaris in elbow region or lower arm

DS750 Lesion in arteria femoralis

DT145 Lesion in blood vessel without indication of body region

DT801 Complication in vessel after infusion, transfusion or injection

DT808 Other complication after infusion, transfusion or injection

DT809 Complication after infusion, transfusion or injection unspecified

DT812K Inadvertent preoperative puncture/lesion of vein or lymph system

DT817 Complication in vessel after intervention unspecified

DT88 Complications with or with surgery

DT888 Complication with surgery or with unspecified treatment, other specified

68229 Inflammation, abscess and acute lymphangioma. brachii and antebrachii

Embolism or thrombosis Embolism or thrombosis Embolism or thrombosis Embolism or thrombosis Embolism or thrombosis Embolism or thrombosis Embolism or thrombosis Embolism or thrombosis Embolism or thrombosis Aneurysm

Aneurysm

Aneurysm

Aneurysm

Aneurysm

Nerve damage

Nerve damage

Arteriovenous fistulas

Other

Other

Other

Other

Other

Other

Other

Other

Other

Other

Other 


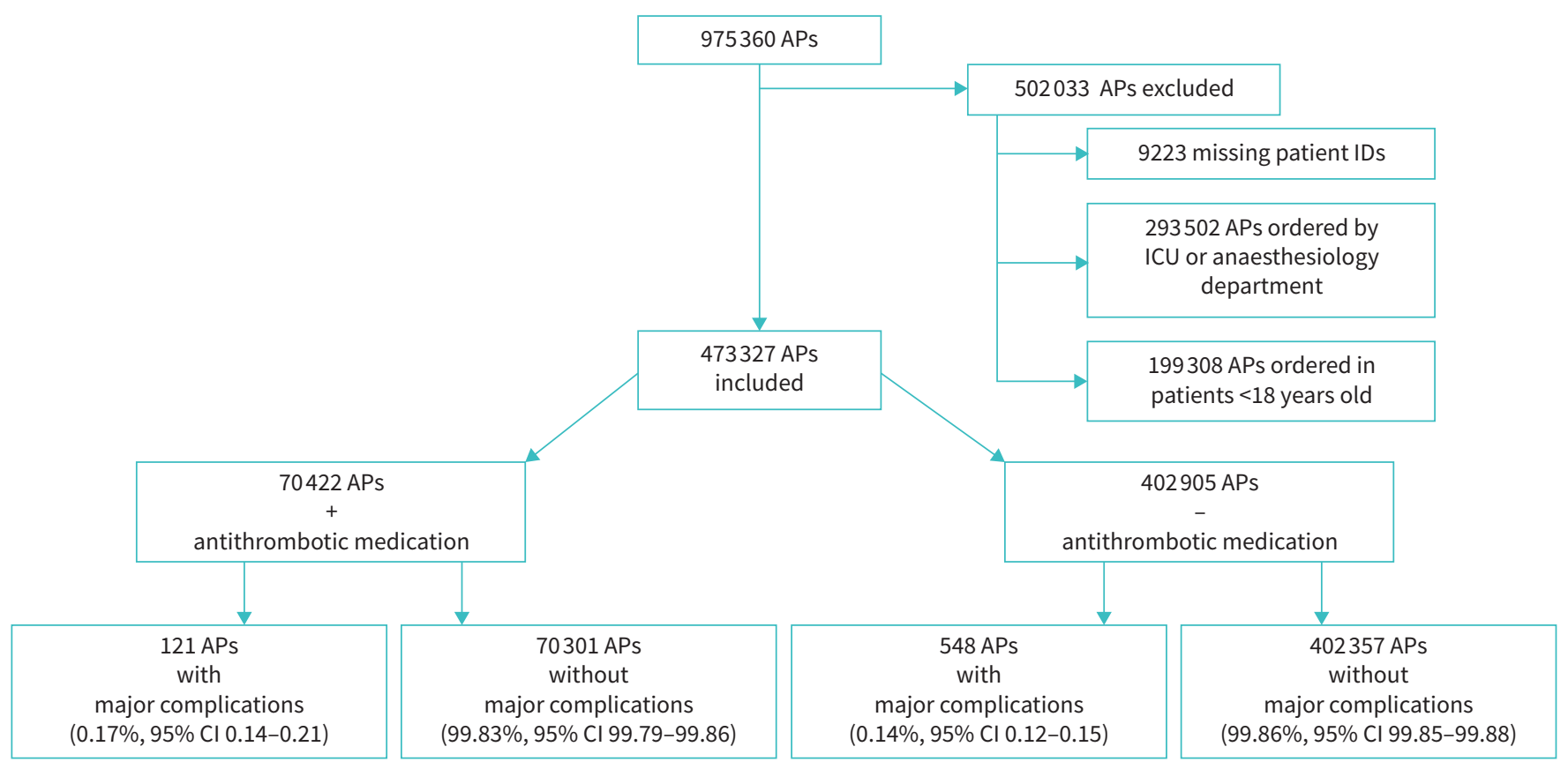

FIGURE 1 Flowchart of major complications in arterial punctures (APs) in patients on and off antithrombotic medication, in three hospitals in Denmark from 1993 to 2013. ICU: intensive care unit.

$51.3 \%$ of the APs were performed in males $(n=242867)$. The median age at the time of the AP was 69 years (IQR 58-77 years). 28.4\% of the APs were performed in patients with a CCI score of $0,45.1 \%$ in patients with a CCI score of $1-2$ and $26.5 \%$ at a CCI score of $\geq 3$ (table 3 ).

\section{Complications}

Major complications occurred after 669 out of 473327 APs (0.14\%, 95\% CI 0.13-0.15). The complications occurred in 303 patients $(0.28 \%$, 95\% CI $0.25-0.31)$. The majority of complications occurred in males $(59.8 \%, n=400)$. The median age at the time of the AP leading to a major complication was 69 years (IQR 61-76). A total of $28.7 \%$ of the APs with a major complication were performed in patients with a CCI score of $0,44.8 \%$ in patients with a CCI score of $1-2$ and $26.5 \%$ at a CCI score of $>3$ (table 4 ).

Figure 2 shows the percentage of APs per year from 1993 to 2013 leading to a major complication, as a total and by sex. The major complication rate tends to show a slight decrease over the years, with the highest rate in 1993.

TABLE 3 Baseline characteristics of patients having an arterial blood gas drawn in the period from January 1 , 1993 to February 25, 2013

\begin{tabular}{|c|c|c|c|c|}
\hline & Total punctures & Age $18-64$ years & Age $65-79$ years & Age $\geqslant 80$ years \\
\hline Total $n$ & 473327 & 177978 & 205228 & 90121 \\
\hline Age years & $69(58-77)$ & $53(43-60)$ & $72(69-76)$ & $84(81-87)$ \\
\hline \multicolumn{5}{|l|}{ Sex } \\
\hline Male & 242867 (51.3) & 95811 (53.8) & 104995 (51.2) & $42061(46.7)$ \\
\hline Female & $230460(48.7)$ & 82167 (46.2) & $100233(48.8)$ & 48060 (53.3) \\
\hline \multicolumn{5}{|c|}{ Charlson Comorbidity Index } \\
\hline 0 & $134514(28.4)$ & 74310 (41.8) & 40733 (19.8) & $19471(21.6)$ \\
\hline 1 & $126142(26.7)$ & 45325 (25.5) & $58337(28.4)$ & $22480(24.9)$ \\
\hline 2 & 87244 (18.4) & 24101 (13.5) & $43246(21.1)$ & $19897(22.1)$ \\
\hline$\geqslant 3$ & $125427(26.5)$ & $50449(17.3)$ & 83863 (26.4) & $30586(30.5)$ \\
\hline
\end{tabular}




\begin{tabular}{|c|c|c|c|c|}
\hline & Total punctures & Age $18-64$ years & Age $65-79$ years & Age $\geqslant 80$ years \\
\hline Total $n$ & 669 & 255 & 300 & 114 \\
\hline Age years & $69(61-76)$ & $55(48-63)$ & $74(68-76)$ & $83(82-87)$ \\
\hline \multicolumn{5}{|l|}{ Sex } \\
\hline Male & $400(59.8)$ & $155(60.8)$ & $192(64.0)$ & $53(46.5)$ \\
\hline Female & $269(40.2)$ & $100(39.2)$ & $108(36.0)$ & $61(53.5)$ \\
\hline \multicolumn{5}{|c|}{ Charlson Comorbidity Index } \\
\hline 0 & $192(28.7)$ & $71(27.8)$ & $92(30.7)$ & $29(25.4)$ \\
\hline 1 & $201(30.0)$ & $95(37.3)$ & $91(30.3)$ & $15(13.2)$ \\
\hline 2 & $99(14.8)$ & $40(15.7)$ & $34(11.3)$ & $25(21.9)$ \\
\hline$\geqslant 3$ & $177(26.5)$ & $49(19.2)$ & $83(27.7)$ & $45(39.5)$ \\
\hline
\end{tabular}

The categories of major complications were embolisms or thrombosis (49.0\%), aneurysms (15.4\%), nerve damage (1.5\%), arteriovenous fistulas ( $0.6 \%$ ) or of another kind (33.5\%), such as lesion of an artery (table 5 ).

\section{Antithrombotic medication}

A total of 70422 APs (14.9\%) were performed in patients who fulfilled a prescription of at least one type of antithrombotic medication (respectively anticoagulant or antiplatelet treatment), within 90 days prior to the AP. As shown in figure 1 and table 6, the complication rates per AP were comparable; respectively $0.14 \%$ (95\% CI 0.12-0.15) in APs in patients without antithrombotic use and $0.17 \%$ (95\% CI $0.14-0.21$ ) in APs in patients with antithrombotic use. We found a statistically significant difference in complication rates in APs in patients on and off antithrombotics $(p=0.02)$. We did not find a statistically significant difference in complication rates when comparing APs in patients using three different medication subgroups: use of one anticoagulant, use of one antithrombotic and use of $\geq 2$ types of antithrombotic medication ( $\mathrm{p}=0.11)$ (table 6).

\section{Risk factors}

We found, when adjusted for age, sex, CCI and use of antithrombotic medication, the only variables associated with an increased risk of developing a major complication are male sex (OR 1.41, 95\% CI 1.20-1.64) and using antithrombotic medication (OR 1.31, 95\% CI 1.07-1.61) (table 7). All other analysed risk factors were not significant. All the possible confounders were predefined as potentially clinically relevant.

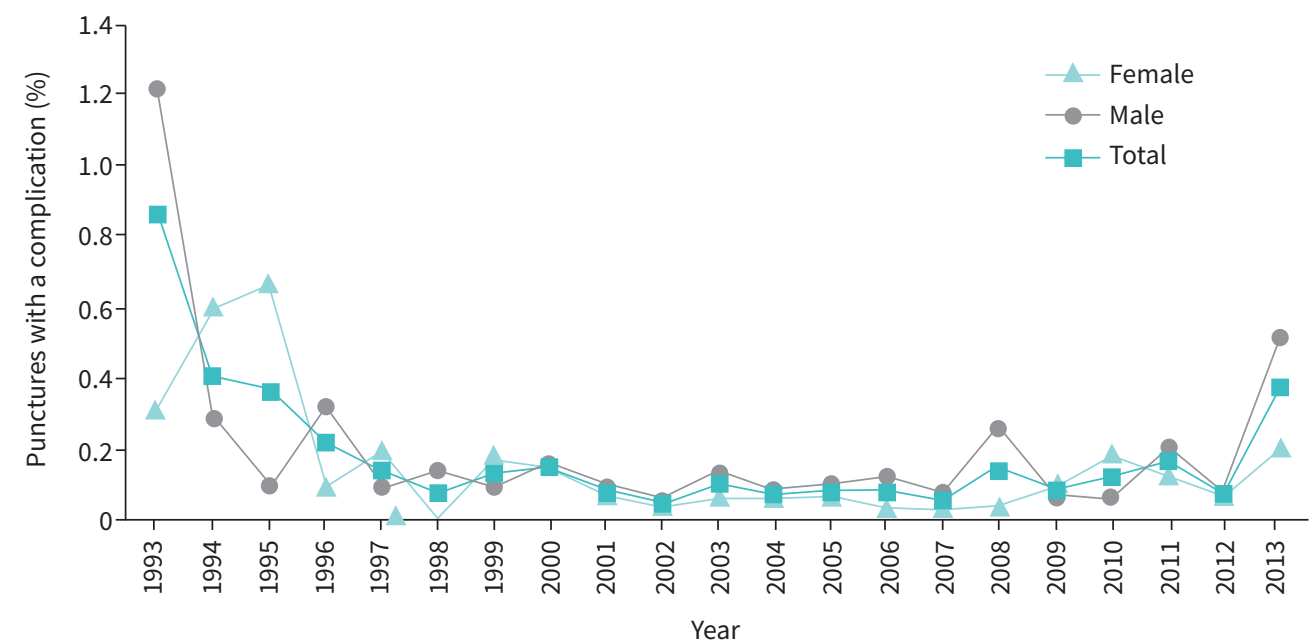

FIGURE 2 Percentage of arterial punctures leading to a major complication, stratified by sex and year in three hospitals in Denmark from 1993 to 2013. 


\section{TABLE 5 Major complications after an arterial puncture for arterial blood gas analysis}

\begin{tabular}{lcc} 
& Total complications $\mathbf{n}$ & \% of total complications $(95 \% \mathrm{Cl})$ \\
\hline Total $\mathbf{n}$ & 669 & 100.0 \\
\hline Embolisms or thrombosis & 328 & $49.0(45.2-52.9)$ \\
Aneurysms & 103 & $15.4(12.7-18.4)$ \\
Nerve damage & 10 & $1.5(0.72-2.7)$ \\
Arteriovenous fistulas & 4 & $0.6(0.16-1.5)$ \\
\hline Other & 224 & $33.5(29.9-37.2)$ \\
\hline
\end{tabular}

\section{Maximum complication rate}

From the 473327 analysed, 1762 APs $(0.37 \%$, 95\% CI $0.36-0.39)$ led to a major complication when calculating the maximum complication rate, an overestimation of the actual major complication rate. In this consideration the complications occurred in 633 patients $(0.58 \%$, 95\% CI 0.53-0.62).

\section{Discussion}

In this multicentre historical cohort study, we analysed 473327 APs and found them to be safe: they have a low major complication rate, with $0.14 \%$ (95\% CI $0.13-0.15)$ of the APs leading to major complications (i.e. embolisms or thrombosis, aneurysms, nerve damage, arteriovenous fistulas or of another kind). Patients on antithrombotics are associated with an increased risk of developing major complications $(0.17 \%, 95 \%$ CI $0.14-0.21)$. The only variables associated with an increased risk of developing a major complication after an AP are male sex and using antithrombotic medication.

This is the first large study on this topic: comparisons with existing studies are restricted since they are relatively small. Previously reported major complication rates were low, although the occasional registered major complications and single case reports show that major complications can occur, which is in accordance with our findings $[9,10,12]$. Research with a database as large as ours allows insight into the actual incidence of these major complications. Women seemed to have a higher complication rate in 1993, but we cannot clarify why. This study additionally provides evidence that patients on antithrombotic therapy have an increased complication rate $(\mathrm{p}=0.02)$. When further stratified by type and number of antithrombotic medication and comparing four groups, no significant difference $(\mathrm{p}=0.11)$ was found. Our findings disagree with the only study we found on this topic which reported that patients on anticoagulants had over four times more complications (both minor and major). That study included punctures for arterial entry, catheterisation and arteriotomy [12]. Concerning the difference in risk related to sex, no similar bibliographic records have been found. IsHII et al. (2012) [9] found no significant difference between complications in males and females; no other studies reported on this outcome variable. This study does not provide an answer to the question why male sex is associated with an increased complication rate. Moreover, the finding that in the period 1993-1994 more complications were recorded in woman was probably due to statistical variation. Our study provides evidence that the number of APs performed in the prior 7 days does not increase the adjusted odds ratio on developing a major complication (OR 0.89, 95\% CI 0.76-1.04). This is in agreement with the only other study on APs reporting on this variable [30].

To establish a causal relationship with major complications as defined here, a different kind of study set-up has to be used. Access to individual medical records would be required to investigate every patient with an AP that presumably led to a complication. This would however not increase the risk estimate. It would only lead to exclusion of events that are, at present, labelled as complications. Moreover, as we did not

TABLE 6 Arterial punctures leading to a major complication in patients on and off antithrombotic medication, further stratified by type and number of antithrombotic medications

\begin{tabular}{lccc} 
& No complications $\mathbf{n}(\%, 95 \% \mathrm{Cl})$ & Complications n (\%, 95\% Cl) & p-value \\
\hline Total $\mathbf{n}$ & $472658(100)$ & $669(100)$ & \\
Antithrombotic medication - & $402357(85.1,85.0-85.2)$ & $548(81.9,78.8-84.8)$ & \\
Antithrombotic medication + & $70301(14.9,14.8-15.0)$ & $121(18.115 .2-21.2)$ & 0.02 \\
1 anticoagulant & $9776(2.1,2.0-2.1)$ & $19(2.8,1.7-4.4)$ & \\
1 antiplatelet & $33501(7.1,7.0-7.2)$ & $58(8.7,6.6-11.1)$ & \\
2 antithrombotics & $27035(5.7,5.7-5.8)$ & $44(6.6,4.8-8.7)$ & 0.11 \\
\hline
\end{tabular}


TABLE 7 Unmatched logistic regression on patients with and without complications

\begin{tabular}{|c|c|c|}
\hline Covariate & Unadjusted odds ratio $(95 \% \mathrm{Cl})$ & Adjusted odds ratio $(95 \% \mathrm{Cl})$ \\
\hline \multicolumn{3}{|l|}{ Sex } \\
\hline Female & & Ref. \\
\hline Male & & $1.41(1.20-1.64)$ \\
\hline \multicolumn{3}{|l|}{ Age } \\
\hline $18-64$ years & & Ref. \\
\hline $65-79$ years & & $1.02(0.86-1.22)$ \\
\hline $80+$ years & & $0.87(0.70-1.10)$ \\
\hline \multicolumn{3}{|l|}{ Charlson Comorbidity Index } \\
\hline 0 & & Ref. \\
\hline 1 & & $1.12(0.92-1.37)$ \\
\hline 2 & & $0.78(0.61-1.00)$ \\
\hline$\geqslant 3$ & & $0.94(0.76-1.16)$ \\
\hline \multicolumn{3}{|l|}{ Antithrombotic medication use } \\
\hline No & Ref. & Ref. \\
\hline Yes & $1.26(1.04-1.54, p=0.02)$ & $1.31(1.07-1.61)$ \\
\hline Arterial puncture in previous 7 days & & $0.89(0.76-1.04)$ \\
\hline
\end{tabular}

have access to the medical records, it was impossible to assess that the procedure did not endanger the lives of the patients to whom it was applied. As far as we can see, there were no life-threatening complications reported. Unfortunately, it is not possible to distinguish between two APs performed within a very short period of time: we simply do not know which one led to the assumed complication. Another challenging task for further research is analysing utilisation patterns of ABGs. A study attempting this topic found $27.6 \%$ of ABGs were ordered as routine, with $79 \%$ of the results being as expected [1]. However, these results led to a change in patient management in only $42 \%$ of the cases. This shows that fewer ABGs can be performed. Providing more insight into these patterns creates clinical awareness of the utilisation of ABGs and could influence clinical decision-making: potentially fewer ABGs could be performed, decreasing risk exposure and costs. Finally, studies on venous blood gas analysis agree that the former in many, but definitely not all, settings can replace ABG analysis. Exploring these different settings could be of interest since venous sampling is executed in many patients anyway and is said to be more convenient: arterial sampling is not always possible, for instance in early stages of resuscitation [31-38]. Moreover, we advise APs to be performed following protocol: work as clean as possible, use the correct equipment and keep the indications and contraindications in mind, such as described by Dev et al. (2011) [39].

\section{Strengths and limitations}

No other study of this size on APs and subsequent complications has ever been undertaken; data were collected in multiple settings over a period of 20 years. The CPR-registry allowed complete follow-up for all patients with a Danish CPR-number. The sample size provided unique evidence about major complications and the rate at which they occur. If there was a clinically significant major complication, it would have been registered. All of these components contribute to the high external validity of our results.

Unfortunately, there are limitations to this historical cohort study. By excluding patients with APs ordered by the anaesthesiology department or ICU, it can be assumed that a healthier patient population was sampled. Since this is a register-based study, only complications that were actually registered could be included: perhaps patients had a major complication after discharge and did not return to the hospital. While we knew that APs have minor complications, using the current set-up, we could not analyse this, as complications not requiring procedures would not be obtainable in the registers. Moreover, it is not clear whether APs and complications were related: we only described the registration of procedures and diagnoses (i.e. a proxy of a major complication) which we assumed were the result of an AP. Possibly complications were registered with multiple codes: one complication could have been followed by several procedures. In that case our analysis made the assumption that one patient had more than one complication, thus leading to an overestimation of the realistic complication rate. Perhaps the way SKS and ICD codes were used and registered could contribute to the reduction in incidence of major complications during the study period. Unfortunately, due to the descriptive and observational nature of this study design, no causal relationship can be described. The chosen cut-off point was 7 days after the AP; if a major complication occurred later, we assumed that the cause was not related to the AP. Naturally it is possible that some of the major complications presented themselves outside our chosen 7-day time-frame; this 
would not say anything about the 7-day complication rate but could be relevant to the overall presumption of the safety of APs. Furthermore, as ICD codes are registered at discharge or at the time of in-hospital transfer, possibly patients with a longer length of stay - presumably the sicker patient population - with a major complication were missed due to the 7-day cut-off point. Also, major complications were subjectively defined; various specialists assessed them with different opinions on what was considered to be a major complication and how likely it was to occur. Owing to agreement by oral consensus by the two clinicians, it was not possible to calculate a Kappa value. If physicians from other specialities performed assessment, it is plausible that the major complication rates could have differed.

\section{Conclusion}

APs for ABG analysis are safe. We found the 7-day major complication rate to be $0.14 \%$ (95\% CI 0.13 0.15). The complications recorded were embolisms or thrombosis $(49.0 \%)$, aneurysms $(15.4 \%)$, nerve damage (1.5\%), arteriovenous fistulas $(0.6 \%)$ or of another kind (33.5\%). Patients who have fulfilled an antithrombotic drug prescription within 90 days before the AP have an increased risk of developing major complications.

Acknowledgements: The authors express their sincere gratitude to Niels Ibsgaard Agerbek from the Center for Clinical Epidemiology and Research Unit of Clinical Epidemiology at the Odense University Hospital, for all the help in constructing the database.

Provenance: Submitted article, peer reviewed.

Availability of data and material: The corresponding author can be contacted about information on how to access any supplemental information such as the study protocol, raw data or programming code, but raw data cannot be exported due to Danish law.

Author contributions: Andreas Alberg-Fløjborg, Marianne Fløjstrup and Daniel Pilsgaard Henriksen contributed substantially to the process of data analysis. Daniel Pilsgaard Henriksen, Bjarke Viberg, Christian Hallenberg, Jes Sanddal Lindholt and Mikkel Brabrand assessed and selected the major complications as described in the methods section. Prabath W.B. Nanayakkara and Mikkel Brabrand gave supervision throughout the entire study. All the authors critically revised the work and gave their final approval of the version to be published.

Conflicts of interest: The authors declare that they have no conflict of interest.

References

1 Melanson SEF, Szymanski T, Rogers SO, et al. Utilization of arterial blood gas measurements in a large tertiary care hospital. Am J Clin Pathol 2007; 127: 604-609.

2 Sado DM, Deakin CD. Local anaesthesia for venous cannulation and arterial blood gas sampling: are doctors using it? J R Soc Med 2005; 98: 158-160.

3 Giner J, Casan P, Belda J, et al. Pain during arterial puncture. Chest 1996; 110: 1443-1445.

4 Hajiseyedjavady $\mathrm{H}$, Saeedi M, Eslami V, et al. Less painful arterial blood gas sampling using jet injection of 2\% lidocaine: a randomized controlled clinical trial. Am J Emerg Med 2012; 30: 1100-1104.

5 Turner JS, Briggs SJ, Springhorn HE, et al. Patients' recollection of intensive care unit experience. Crit Care Med 1990; 18: 966-968.

6 Roberts, JR, Custalow, CB, Thomsen, TW, et al. Arterial puncture and cannulation. In: Kim HT, ed. Roberts and Hedges' Clinical Procedures in Emergency Medicine. Philadelphia, PA: Saunders, an imprint of Elsevier Inc, 2014; pp. 368-384.

7 Walensi M, Elsner A. Arterieller zugang-indikationen, techniken, komplikationen. Dtsch Med Wochenschr 2016; 141: $1630-1635$.

8 Fleming WH, Bowen JC. Complications of arterial puncture. Mil Med 1974; 139: 307-308.

9 Ishii S, Miyajima M, Shishido F, et al. Hematoma after femoral arterial blood sampling. J Comput Assist Tomogr 2012; 36: 257-260.

10 Okeson GC, Wulbrecht PH. The safety of brachial artery puncture for arterial blood sampling. Chest 1998; 114: 748-751.

11 Tong Z, Gu Y, Guo L, et al. An analysis of complications of brachial and axillary artery punctures. Am Surg 2016; 82: 1250-1256.

12 Mortensen JD. Clinical sequelae from arterial needle puncture, cannulation, and incision. Circulation 1967; 35 1118-1123.

13 Ara C, Coban S, Isik B, et al. Small bowel perforation after drawing a blood sample in the femoral artery: a case report. Ulus Travma Acil Cerrahi Derg 2010; 16: 275-276. 

intensive care setting. Chest 1995; 108: 578-580.

15 Marshall G, Edelstein G, Hirshman CA. Median nerve compression following radial arterial puncture. Anesth Analg 1980; 59: 953-954.

16 Matsagas MI, Mitsis M, Rigopoulos C, et al. A large radial artery false aneurysm after repeated arterial punctures, causing compartment syndrome of the forearm. Intensive Care Med 2003; $29: 1032$.

17 Matthews JI, Gibbons RB. Embolization complicating radial artery puncture. Ann Intern Med 1971; 75: 87-88.

18 Patel KN, Gandhi SP, Sutariya HC. Radial artery pseudoaneurysm: a rare complication after a single arterial puncture for blood-gas analysis. Indian J Crit Care Med 2016; 20: 622-626.

19 Russo G, Cherubini A, Fisicaro M, et al. Radial pseudoaneurysm after a puncture for blood gas analysis. Am $J$ Emerg Med 2016; 34: 757.e1-757.e2.

20 Malík J, Holaj R, Krupičková Z, et al. Arteriovenous fistula after femoral artery puncture leading to pulmonary edema: the role of ultrasonography. Prague Me Rep 2012; 113: 49-52.

21 Berger A. Brachial artery puncture: the need for caution. J Fam Pract 1989; 28: 720-722.

22 Neviaser RJ, Adams JP, May GI. Complications of arterial puncture in anticoagulated patients. JBJS 1976; 58: 218-220.

23 Benchimol El, Smeeth L, Guttmann A, et al. The REporting of studies Conducted using Observational Routinely-collected health Data (RECORD) statement. PLoS Med 2015; 12: e1001885.

24 Quan H, Li B, Couris CM, et al. Updating and validating the Charlson comorbidity index and score for risk adjustment in hospital discharge abstracts using data from 6 countries. Am J Epidemiol 2011; 173: 676-682.

25 Radovanovic D, Seifert B, Urban P, et al. Validity of Charlson Comorbidity Index in patients hospitalised with acute coronary syndrome. Insights from the nationwide AMIS Plus registry 2002-2012. Heart 2014; 100: 288-294.

26 Schmidt M, Pedersen L, Sørensen HT. The Danish civil registration system as a tool in epidemiology. Eur $J$ Epidemiol 2014; 29: 541-549.

27 Schmidt M, Schmidt SAJ, Sandegaard JL, et al. The Danish national patient registry: a review of content, data quality, and research potential. Clin Epidemiol 2015; 7: 449-490.

28 Bjerrum L, Bergman U. Wide variation in the number of different drugs prescribed by general practitioners. A prescription database study. Scand J Prim Health Care 2000; 18: 94-98.

29 Jarlbaek L, Andersen M, Kragstrup J, et al. Cancer patients' share in a population's use of opioids. A linkage study between a prescription database and the Danish Cancer Registry. J Pain Symptom Manage 2004; 27: 36-43.

30 Gillies IDS, Morgan M, Sykes MK, et al. The nature and incidence of complications of peripheral arterial puncture. Anaesthesia 1979; 34: 506-509.

31 Brandenburg MA, Dire DJ. Comparison of arterial and venous blood gas values in the initial emergency department evaluation of patients with diabetic ketoacidosis. Ymem 1998; 31: 459-465.

32 Burri E, Potocki M, Drexler B, et al. Value of arterial blood gas analysis in patients with acute dyspnea: an observational study. Crit Care 2011; 15: R145.

33 Malatesha G, Singh NK, Bharija A, et al. Comparison of arterial and venous $\mathrm{pH}$, bicarbonate, $\mathrm{PCO}_{2}$ and $\mathrm{PO}_{2}$ in initial emergency department assessment. Emerg Med J 2007; 24: 569-571.

34 Middleton P, Kelly AM, Brown J, et al. Agreement between arterial and central venous values for $\mathrm{pH}$, bicarbonate, base excess, and lactate. Emerg Med J 2006; 23: 622-624.

35 Kelly A-M. Review article: Can venous blood gas analysis replace arterial in emergency medical care. Emerg Med Australas 2010; 22: 493-498.

36 Rudkin SE, Kahn CA, Oman JA, et al. Prospective correlation of arterial vs venous blood gas measurements in trauma patients. Am J Emerg Med 2012; 30: 1371-1377.

37 Treger R, Pirouz S, Kamangar N, et al. Agreement between central venous and arterial blood gas measurements in the intensive care unit. Clin J Am Soc Nephrol 2010; 5: 390-394.

38 Walkey AJ, Farber HW, O'Donnell C, et al. The accuracy of the central venous blood gas for acid-base monitoring. J Intensive Care Med 2010; 25: 104-110.

39 Dev SP, Hillmer MD, Ferri M. Arterial puncture for blood gas analysis. N Engl J Med 2011; 364 : e7. 